\title{
An Alternate Version of Pirogov-Sinai Theory
}

\author{
Miloš Zahradník \\ Faculty of Mathematics and Physics, Charles University, Prague, Czechoslovakia
}

\begin{abstract}
A new approach to the Pirogov-Sinai theory of phase transitions is developed, not employing the contour models with a parameter. The completeness of the phase diagram is proven.
\end{abstract}

\section{Introduction}

The aim of the following note is twofold.

i) The theory of Pirogov and Sinai describing the behaviour of phase diagrams of lattice models with discrete spins is based on the notion of a contour model and a contour model with a parameter. The notion of a contour model goes back to Minlos and Sinai's detailed study of the Ising model [1].

If one is interested in the investigation of the behaviour of the point of the maximal number of phases only, there is no need for introducing the models with parameter in the Pirogov-Sinai (PS) theory. This was, historically, the first case studied, but it had many of the essential features of the theory.

It is the aim of the first part of this note to show that even the full phase diagram can be constructed without the use of models with a parameter. Instead, we will introduce an auxiliary "metastable" contour model, not appearing in the usual PS theory. It seems that this approach is (intuitively, at least) simpler than the original approach [2]. It gives some additional information, too.

ii) There are some results of Martirosian (see e.g. [4]) concerning the completeness of the phase diagram constructed by the PS method. The full answer is given here. The proof is simple and uses the same tools which were developed in i). In particular, we emphasise the technical main lemma which is crucial in the proofs of both parts of this paper.

The organization of the paper is as follows:

Section 1 is devoted to the questions mentioned in i). We suppose some familiarity with PS ideas and notions like contours, contour models etc. For these notions which are only sketched the standard reference is [2] or [3], but no technical details of [2] are used in this paper. 
The technical lemmas of this paper are collected in Sect. 2. Here, some familiarity with the cluster expansion method is useful, as well as with the notion of a contour (or polymer) model. Basic results of this method (the expansion of the polymer partition function into the volume and boundary part, in particular) are stated without proof. As a byproduct of the cluster expansion method, we formulate also our main lemma.

Finally, the proof of the completeness is given in Sect. 3.

We do not formulate any results about piecewise analyticity of the phase diagram. Also, we omit a possible generalization to the continuous spin models. This will be a subject of forthcoming papers.

\section{Construction of the Phase Diagram}

\subsection{Preliminary Notions}

Let $\mathbb{Z}^{v}$ be a $v$-dimensional lattice ( $v \geqq 2$ ), and let $S$ be a finite set (of "spins"). For any $\Lambda \subset \mathbb{Z}^{v}$, denote by $S^{\Lambda}$ the set of all configurations on $\Lambda$. Suppose that some family $\left\{\Phi_{A}\right\}$ of finite interactions (i.e. functions on $S^{A}$ ) is given, invariant with respect to shifts in $\mathbb{Z}^{v}$ and with a finite interaction radius $r$ (i.e. such that $\Phi_{A} \equiv 0$ if $\operatorname{diam} A>r$ ).

Note. We consider, here and everywhere in the following, the norm

$$
\|t\|=\sum_{i=1}^{v}\left|t_{i}\right|, \quad t \in \mathbb{Z}^{v} .
$$

Given $\Lambda \subset \mathbb{Z}^{v}$, we denote by $\partial \Lambda$ the set

$$
\partial \Lambda=\left\{t \in \Lambda: \operatorname{dist}\left(t, \Lambda^{c}\right)=1\right\} .
$$

For each finite $\Lambda \subset \mathbb{Z}^{v}$ and each $x \in S^{\mathbb{Z}^{v}}$, denote by $x_{\Lambda}$ the restriction of $x$ on $\Lambda$. Define the hamiltonians

$$
\begin{gathered}
H\left(x_{A}\right)=\sum_{A \subset A} \Phi_{A}\left(x_{A}\right), \\
H\left(x_{\Lambda} / x_{\Lambda^{c}}\right)=\sum_{A \not \subset \Lambda^{c}} \Phi_{A}\left(x_{A}\right) .
\end{gathered}
$$

Define the mean energy of any periodical configuration $x \in S^{\mathbb{Z}^{*}}$ :

$$
e(x)=\lim _{\Lambda \uparrow \mathbb{Z}^{v}}|\Lambda|^{-1} H\left(x_{\Lambda}\right)
$$

(the limit is taken in the Van Hove sense).

Given $q \in S$, denote by $x_{q}$ the configuration $\left\{x_{t} \equiv q, t \in \mathbb{Z}^{v}\right\}$ and denote by $e_{q}=e\left(x_{q}\right)$. Note that $e_{q}$ can be expressed by the formula

$$
e_{q}=\sum_{A: A \ni 0}|A|^{-1} \Phi_{A}\left(\left(x_{q}\right)_{A}\right) \text {. }
$$

\subsection{Ground States, Boundaries, Contours}

Fix some family $\left\{x_{q}, q \in Q\right\}, Q \subset S$ of constant configurations. Call these fixed configurations ground states of our model. 
Note. The connection with the usual meaning of the notion of a ground state will be seen in Sect. 1.4, where an additional condition (Peierls condition) will be imposed on $x_{q}, q \in Q$. Typically, in the following, $H$ will be a perturbation of some hamiltonian $H_{0}$ such that $x_{q}, q \in Q$ are exactly the ground states (in the usual meaning of PS theory) of $H_{0}$.

Say that a point $t \in \mathbb{Z}^{v}$ is a $q$-correct point of a configuration $x \in S^{\mathbb{Z}^{v}}$ if for each $s \in \mathbb{Z}^{v}$ such that $\|t-s\| \leqq r, x_{s}$ has a constant value $x_{s}=q \in Q$. Say that $t \in \mathbb{Z}^{v}$ is a boundary point of a configuration $x$ if it is incorrect for each "ground" $q \in Q$.

A boundary of a configuration $x$ is defined as the union of all boundary points of $x$. It will be denoted as $B(x)$. A restriction of $x$ to any finite component of $B(x)$ will be called a contour (of a configuration $x$ ), and this component will be called the support of this contour.

Having some contour $\Gamma$, a component of $(\operatorname{supp} \Gamma)^{c}$ will be called a $q$-component if the neighboring spins of $\Gamma$ have the value $q$. If the exterior component of $(\operatorname{supp} \Gamma)^{c}$ is a $q$-component, then $\Gamma$ will be called $q$-contour and denoted by $\Gamma^{q}$. For any $q^{\prime} \in Q$ denote by $\operatorname{int}_{q^{\prime}} \Gamma$ the union of all interior $q^{\prime}$-components of $(\operatorname{supp} \Gamma)^{c}$. Put

$$
\begin{aligned}
& \operatorname{int} \Gamma=\bigcup_{q^{\prime} \in Q} \operatorname{int}_{q^{\prime}} \Gamma, \\
& V(\Gamma)=\operatorname{supp} \Gamma \cup \operatorname{int} \Gamma, \\
& \operatorname{ext} \Gamma=(V(\Gamma))^{c} .
\end{aligned}
$$

\subsection{Contour Hamiltonians, Expression of $H\left(x_{\Lambda} \mid x_{A^{c}}\right)$}

Let $\Gamma=\Gamma^{q}$ be a contour of some configuration $x \in S^{\mathbb{Z}^{v}}$. Put

$$
\Phi(\Gamma)=\sum_{A \subset \mathbb{Z}^{v}}\left(|A|^{-1}|A \cap \operatorname{supp} \Gamma| \Phi_{A}\left(x_{A}\right)\right)-e_{q}|\operatorname{supp} \Gamma| .
$$

Note. This value obviously does not depend on the choice of $x$.

Proposition 1. Let $\Lambda \subset \mathbb{Z}^{v}$ be finite, and let $x \in S^{\mathbb{Z}^{v}}$ be some configuration such that all its contours satisfy the condition $\operatorname{supp} \Gamma \subset \Lambda$. Denote by $\Lambda_{q}$ the set of all $t \in \Lambda$ such that either $t$ is a q-correct point of $x$ or $t \in \operatorname{supp} \Gamma^{q}$ for some contour $\Gamma^{q}$ of $x$. Then using the notations (1.4), (1.6),

$$
H\left(x_{\Lambda} \mid x_{\Lambda^{c}}\right)=\sum_{\Gamma} \Phi(\Gamma)+\sum_{q} e_{q}\left|\Lambda_{q}\right|+\sum_{A \not \subset \Lambda} \frac{\left|A \cap \Lambda^{c}\right|}{|A|} \Phi_{A}\left(x_{A}\right) .
$$

Proof. This is obvious if we substitute

$$
\Phi_{A}\left(x_{A}\right)=\sum_{t \in A}|A|^{-1} \Phi_{A}\left(x_{A}\right)
$$

into (1.2) and rearrange, using (1.6) and (1.4), the terms thus obtained.

Note. Obviously, the last term of (1.7) does not depend on $x_{A}$. Therefore, discarding this term from $H\left(x_{\Lambda} \mid x_{\Lambda^{c}}\right)$ does not effect the conditional Gibbs densities. Because we will consider throughout Sect. 1 only such restricted ensembles where (1.7) is applicable, we will replace our original hamiltonians by modified ones,

$$
H\left(x_{\Lambda} \mid x_{\Lambda^{c}}\right)=\sum_{\Gamma} \Phi(\Gamma)+\sum_{q} e_{q}\left|\Lambda_{q}\right| .
$$




\subsection{Peierls (Gertzik-Pirogov-Sinai) Condition}

Suppose that there is a sufficiently large $\tau>0$ such that

$$
\Phi(\Gamma)>\tau|\operatorname{supp} \Gamma|
$$

for each contour $\Gamma$.

\subsection{Definitions of Various Partition Functions}

Given a contour $\Gamma=\Gamma^{q}$, denote by $X(\Gamma)$ the set of all configurations $x_{V}$ on $V(\Gamma)$ which satisfy the condition that being extended by $q$ to the whole $\mathbb{Z}^{v}$, they have $\Gamma$ as one of its contour. Further denote by $X_{\text {red }}(\Gamma)$ the set of all configurations $x_{V}$ on $V(\Gamma)$ which satisfy the condition that after extending them by $q$ to the whole $\mathbb{Z}^{v}$, each contour of the extended configuration has a distance at least 2 from $\operatorname{supp} \Gamma$. Define the "crystallic" partition functions

$$
\mathbf{Z}(\Gamma, H)=\sum_{x_{V} \in X(\Gamma)} \exp \left(-H\left(x_{V} \mid\left(x_{q}\right)_{V(\Gamma)^{c}}\right),\right.
$$

and also the partition functions

$$
\mathbf{Z}\left(\Gamma_{\text {red }}, H\right)=\sum_{x_{V} \in X_{\text {red }}(\Gamma)} \exp \left(-H\left(x_{V} \mid\left(x_{q}\right)_{V(\Gamma)^{c}}\right) .\right.
$$

For any finite $\Lambda \subset \mathbb{Z}^{v}$, denote by $X_{q}(\Lambda)$ the set of all configurations $x_{\Lambda}$ on $\Lambda$ which, being extended by $q$ to the whole $\mathbb{Z}^{v}$ satisfy the condition $\operatorname{dist}\left(V(\Gamma), \Lambda^{c}\right) \geqq 2$ for each contour of the extended configuration. Define the "diluted" partition functions

$$
\mathbf{Z}_{q}(\Lambda, H)=\sum_{x_{\Lambda} \in X_{q}(\Lambda)} \exp \left(-H\left(x_{\Lambda} \mid\left(x_{q}\right)_{\Lambda^{c}}\right) .\right.
$$

Note. i) The hamiltonians $H((\cdot) \mid(\cdot))$ are expressed by $(1.8)$ everywhere.

ii) We emphasise that in the definition of $X_{q}(\Lambda)$ we used the condition

$$
\operatorname{dist}\left(V(\Gamma), \Lambda^{c}\right) \geqq 2
$$

[and not $\operatorname{dist}\left(\operatorname{supp} \Gamma, \Lambda^{c}\right) \geqq 2$ for example]. Note, however, that if $\Lambda$ has simple connected components (this holds e.g. if $\Lambda=\operatorname{int}_{q} \Gamma$ for some $\Gamma$ and $q$ ) then the condition $\operatorname{dist}\left(\operatorname{supp} \Gamma, \Lambda^{c}\right) \geqq 2$ implies the condition (1.13).

\subsection{Contour Functional and Contour Model}

Define a contour functional

$$
\Psi(\Gamma)=\log \mathbf{Z}\left(\Gamma_{\text {red }}, H\right)-\log \mathbf{Z}(\Gamma, H)
$$

and denote by

$$
\mathbf{Z}_{q}(\Lambda, \Psi)=\sum_{\left\{\Gamma_{i}^{q}\right\}} \exp \left(-\sum_{i} \Psi\left(\Gamma_{i}^{q}\right)\right)
$$

where the sum is taken over all families of contours $\left\{\Gamma_{i}^{q}\right\}$ such that (1.13) is satisfied for each $\Gamma_{i}^{q}$ and moreover

$$
\operatorname{dist}\left(\operatorname{supp} \Gamma_{i}^{q}, \operatorname{supp} \Gamma_{i^{\prime}}^{q}\right) \geqq 2 \quad \text { whenever } \quad i \neq i^{\prime} .
$$


Define also the "crystallic" partition function of the contour ensemble [compare $(1.10)$ !]

$$
\mathbf{Z}\left(\Gamma^{q}, \Psi\right)=\sum_{\left\{\Gamma_{i}^{q}\right\}} \exp -\left(\sum_{i} \Psi\left(\Gamma_{i}^{q}\right)\right),
$$

where the sum is taken over all families of contours $\left\{\Gamma_{i}^{q}\right\} \ni \Gamma^{q}$ satisfying (1.16) and such that $\operatorname{supp} \Gamma_{i}^{q} \subset V\left(\Gamma^{q}\right)$ for each $i[$ notice that $\operatorname{supp} \tilde{\Gamma} \subset V(\Gamma) \Rightarrow V(\tilde{\Gamma}) \subset V(\Gamma)$ because of the note below (1.13)].

Proposition. Let $\mathfrak{A}$ be a class of contours (e.g. a class of all contours - another concrete choice of $\mathfrak{A}$ will be specified below). Denote by $Z^{\mathfrak{A}}$ the partition functions of the type (1.12), (1.15) but with an additional restriction that only those $x_{A}$ respectively $\left\{\Gamma_{i}^{q}\right\}$ are accounted in the sum whose external contours belong to the class $\mathfrak{A}$. Then

$$
\mathbf{Z}_{q}^{\mathfrak{Q}}(\Lambda, H)=\exp \left(-e_{q}|\Lambda|\right) \mathbf{Z}_{q}^{\mathfrak{M}}(\Lambda, \Psi) .
$$

Also, for any contour $\Gamma^{q}$,

$$
\mathbf{Z}\left(\Gamma^{q}, H\right)=\exp \left(-e_{q} \mid V\left(\Gamma^{q}\right)\right) \mathbf{Z}\left(\Gamma^{q}, \Psi\right) .
$$

Proof. Define the level of any contour $\Gamma$ as a maximal number $n$ such that there is some sequence of contours $\Gamma_{1} \equiv \Gamma, \Gamma_{2}, \ldots, \Gamma_{n}$ such that $\operatorname{supp} \Gamma_{i+1} \operatorname{Cint} \Gamma_{i}$. Define the level of $\Lambda \subset \mathbb{Z}^{v}$ as a maximal level of any contour $\Gamma$ such that $V(\Gamma) \subset \Lambda$.

Proceed now by the induction on the level of $\Gamma$ respectively $\Lambda$. Denote by $\operatorname{EXT}_{q}^{\mathfrak{Q I}}(\Lambda)$ the family of all systems of contours $\left\{\Gamma_{i}^{q} \in \mathfrak{U}\right\}$ satisfying (1.16) such that $V\left(\Gamma_{i}^{q}\right)$ are mutually disjoint and $\operatorname{dist}\left(V\left(\Gamma_{i}^{q}\right), \Lambda^{c}\right) \geqq 2$ (三external contour systems). Notice the relations

$$
\mathbf{Z}_{q}^{\mathfrak{I}}(\Lambda, H)=\sum_{\operatorname{Ex} T_{q}^{\mathbb{u}}(\Lambda)} \exp \left(-e_{q}\left|\operatorname{ext}\left\{\Gamma_{i}^{q}\right\}\right|\right) \prod_{i} \mathbf{Z}\left(\Gamma_{i}^{q}, H\right),
$$

and

$$
\mathbf{Z}_{q}^{\mathfrak{q}}(\Lambda, \Psi)=\sum_{\operatorname{ExT}_{q}^{q}(\Lambda)} \prod_{i} \mathbf{Z}\left(\Gamma_{i}^{q}, \Psi\right)
$$

where $\operatorname{ext}\left\{\Gamma_{i}^{q}\right\}=\Lambda \bigcup_{i} V\left(\Gamma_{i}^{q}\right)$. Thus (1.19) implies (1.18) for a given level. Conversely, using the very definition of $\Psi(\Gamma)$ [see (1.14)], we get

$$
\begin{aligned}
\mathbf{Z}\left(\Gamma^{q}, H\right) & =\exp \left(-\Psi\left(\Gamma^{q}\right)\right) \mathbf{Z}\left(\Gamma_{\text {red }}^{q}, H\right) \\
& =\exp \left(-\Psi\left(\Gamma^{q}\right)-e_{q}\left|\operatorname{supp} \Gamma^{q}\right|\right) \mathbf{Z}_{q}\left(\operatorname{int} \Gamma^{q}, H\right) \\
& =\exp \left(-\Psi\left(\Gamma^{q}\right)-e_{q}\left|V\left(\Gamma^{q}\right)\right|\right) \mathbf{Z}_{q}\left(\operatorname{int} \Gamma^{q}, \Psi\right) \\
& =\exp \left(-e_{q}\left|V\left(\Gamma^{q}\right)\right|\right) \mathbf{Z}\left(\Gamma^{q}, \Psi\right),
\end{aligned}
$$

which gives (1.19) for the next greater level, q.e.d.

\subsection{Truncated Contour Functional and Truncated Contour Model}

The reason for introducing the contour models is that they can be studied, under the conditions of the type (1.9), by powerful methods of the cluster expansion. But, of course, we cannot expect conditions of this type to hold in the case of functionals $\Psi(\Gamma)$ (in general). 
We choose the following method: we construct an artificial "metastable" contour model where "unstable" contours (i.e. such contours for which the condition of Peierls is violated - see Definition 2) are "stabilized" by fixing their energy at a sufficient level - see Definition 1). Later it will be proved that for some distinguished values of $q$ ("stable" $q$ ), such artificial contour models are equal to the usual contour models and therefore describe some "stable" phase.

Definition 1. A truncated contour functional is defined as

$$
\Psi^{\prime}(\Gamma)=\max \left(\Psi(\Gamma), \frac{\tau}{3}|\operatorname{supp} \Gamma|\right) .
$$

Definition 2. A contour $\Gamma$ is called stable if

$$
\Psi(\Gamma) \geqq \frac{\tau}{3}|\operatorname{supp} \Gamma|
$$

[i.e. if $\left.\Psi^{\prime}(\Gamma)=\Psi(\Gamma)\right]$.

Definition 3. Replacing $\Psi$ by a contour functional $\Psi^{\prime}$, we define the truncated partition functions

$$
\mathbf{Z}_{q}\left(\Lambda, \Psi^{\prime}\right), \quad \mathbf{Z}_{q}^{\mathfrak{q}}\left(\Lambda, \Psi^{\prime}\right), \quad \mathbf{Z}\left(\Gamma^{q}, \Psi^{\prime}\right)
$$

quite analogously as in (1.15), (1.17), Proposition 1.6.

Definition 4. Define the free energy of the truncated model

$$
S_{q}=\lim _{\Lambda \ngtr \mathbb{Z}^{v}}|\Lambda|^{-1} \log \mathbf{Z}_{q}\left(\Lambda, \Psi^{\prime}\right)
$$

(see Sect. 2.1). Define also the following quantities:

$$
\begin{aligned}
& h_{q}=e_{q}-s_{q}[\operatorname{see}(1.4)] \\
& h_{0}=\min \left\{h_{q}\right\} \\
& a_{q}=h_{q}-h_{0} \\
& a=\min \left\{a_{q}: a_{q} \neq 0\right\} .
\end{aligned}
$$

Definition 5. If $a_{q}=0, q$ is called stable.

The main result of Sect. 1 is the following statement:

Theorem. i) Nonstable contours satisfy the inequality

$$
a_{q}\left|\operatorname{int} \Gamma^{q}\right| \geqq \frac{\tau}{3}\left|\operatorname{supp} \Gamma^{q}\right| .
$$

In particular, if $q$ is stable, then all the contours $\Gamma^{q}$ are stable and $\Psi^{\prime}\left(\Gamma^{q}\right)=\Psi\left(\Gamma^{q}\right)$ for each contour $\Gamma^{q}$. Further, there is some universal constant $C$, such that for each finite $\Lambda \subset \mathbb{Z}^{v}$, the following estimates hold:

$$
\begin{array}{ll}
\text { ii) } & \mathbf{Z}_{q}(\Lambda, H) \geqq \exp \left(-h_{q}|\Lambda|\right) \exp \left(-C\left|\partial \Lambda^{c}\right|\right), \\
\text { iii) } & \mathbf{Z}_{q}(\Lambda, H) \leqq \exp \left(-h_{0}|\Lambda|\right) \exp \left(C\left|\partial \Lambda^{c}\right|\right) .
\end{array}
$$

The constant $C$ can be chosen as $C=2 C_{(2.5)}$ (where $C_{(2.5)}$ is from (2.5)). 
Corollary. For any stable $q$ we can construct a Gibbs state which is a perturbation of the $q^{\text {th }}$ ground state, in the sense described in [2], such that the statistical properties of its external contour systems are the same as in the contour model with a functional $\Psi\left(\Gamma^{q}\right)$. Thus, for each stable $q$ we obtain a distinct phase. The behaviour of the phase picture thus constructed will be studied in Sect. 1.10.

We omit the proof of the Corollary, as it uses some standard arguments of the theory of limit Gibbs states (see [2]). It is based on Proposition 1.6.

\subsection{Proof of Theorem 1.7}

We will proceed again by the induction over the levels of $\Lambda, \Gamma$. The induction step for iii) uses substantially the main lemma of 2.2 .

Proof of ii). By Proposition 1.6,

$$
\mathbf{Z}_{q}(\Lambda, H)=\exp \left(-e_{q}|\Lambda|\right) \mathbf{Z}_{q}(\Lambda, \Psi) .
$$

Because trivially $\mathbf{Z}_{q}(\Lambda, \Psi) \geqq \mathbf{Z}_{q}\left(\Lambda, \Psi^{\prime}\right)$, and

$$
\mathbf{Z}_{q}(\Lambda, \Psi) \geqq \exp \left(s_{q}|\Lambda|\right) \exp \left(-C\left|\partial \Lambda^{c}\right|\right)
$$

by (2.5), we get the desired relation (1.27), with $C=C_{(2.5)}$.

Proof of i). We use the induction argument for iii). Both i) and iii) are trivial if $\Gamma$ respectively $\Lambda$ has level 0 (for $\tau$ sufficiently large). Nonstability means, by (1.23), that

$$
\mathbf{Z}\left(\Gamma^{q}, H\right) \geqq \exp \left(-\frac{\tau}{3}\left|\operatorname{supp} \Gamma^{q}\right|\right) \mathbf{Z}\left(\Gamma_{\text {red }}^{q}, H\right) .
$$

On the other hand, by the induction assumption for iii) we get

$$
\mathbf{Z}\left(\Gamma^{q}, H\right) \leqq \exp \left(-\Phi\left(\Gamma^{q}\right)-e_{q}\left|\operatorname{supp} \Gamma^{q}\right|-h_{0}\left|\operatorname{int} \Gamma^{q}\right|+2 C\left|\operatorname{supp} \Gamma^{q}\right|\right)
$$

[because $\left.\operatorname{supp} \Gamma^{q} \supset \partial\left(\operatorname{int} \Gamma^{q}\right)^{c}\right]$, with $C=C_{(2.5)}$.

By Proposition 1.6 and by (2.5),

$$
\begin{aligned}
\mathbf{Z}\left(\Gamma_{\text {red }}^{q}, H\right) & \geqq \exp \left(-e_{q}\left|V\left(\Gamma^{q}\right)\right|\right) \mathbf{Z}\left(\Gamma_{\text {red }}^{q}, \Psi^{\prime}\right) \\
& \geqq \exp \left(-h_{q}\left|\operatorname{int} \Gamma^{\alpha}\right|-C\left|\operatorname{supp} \Gamma^{q}\right|-e_{q}\left|\operatorname{supp} \Gamma^{q}\right|\right),
\end{aligned}
$$

where $C=C_{(2.5)}$.

If we combine the relations (1.30), (1.31), (1.32) we obtain

$$
a_{q}\left|\operatorname{int} \Gamma^{q}\right| \geqq \Phi\left(\Gamma^{q}\right)-\frac{\tau}{3}\left|\operatorname{supp} \Gamma^{q}\right|-3 C\left|\operatorname{supp} \Gamma^{q}\right| \geqq\left(\frac{2 \tau}{3}-3 C\right)\left|\operatorname{supp} \Gamma^{q}\right|,
$$

with $C=C_{(2.5)}$, but this is (1.26) for a large $\tau$. q.e.d.

Proof of iii). This is the main step of the proof. To have some idea notice that iii) follows from (1.18) and (2.5) whenever all contours $\Gamma_{i}^{q}$ in (1.5) are stable. We need, therefore, some method of handling the unstable contours.

The inconvenient property of the notion of unstable contour is that it is not hereditary with respect to the inclusion $\operatorname{supp} \Gamma^{q} \operatorname{Cint} \widetilde{\Gamma}^{q}:$ if $\Gamma^{q}$ is nonstable then $\widetilde{\Gamma}^{q}$ may be stable. 
We introduce the following important notion:

Definition 1. Say that a contour $\Gamma^{q}$ is small if it is hereditary stable, i.e. if there is no unstable $\widetilde{\Gamma}^{q}$ such that $\operatorname{supp} \tilde{\Gamma}^{q} \mathrm{Cint} \Gamma^{q}$. Say that $\Gamma^{q}$ is large if it is not small.

Notation. Recall that in the definition of $\mathbf{Z}^{\mathfrak{I}}$ we fixed some family of contours $\mathfrak{A}$, not specified until now. Assume, in the rest of Sect. 1, that $\mathfrak{U}$ is the family of all small contours.

Given $x_{\Lambda} \in X_{q}(\Lambda)$, fix all its external large contours [i.e. external contours of the configuration $x_{\Lambda} \cup\left(x_{q}\right)_{\Lambda^{c}}$ - we recall the assumption (1.13) - which are large]. Denote by $\left\{\Gamma_{\text {ext } i}^{q}\right\}$ the collection of all external large contours of $x_{\Lambda}$. Denote by

$$
\operatorname{ext} \equiv \operatorname{ext} x_{\Lambda}=\Lambda \backslash \bigcup_{i} V\left(\Gamma_{\mathrm{ext} i}^{q}\right)
$$

Denote by $W_{\tilde{q}}, \tilde{q} \in Q$ the union of the interior $\tilde{q}$-components of the set $\Lambda \backslash \bigcup_{i} \operatorname{supp}\left(\Gamma_{\mathrm{ext} i}^{q}\right)$. Fix, for a moment, the family of external large contours $\left\{\Gamma_{\mathrm{ext} i}^{q}\right\}$. To determine the whole configuration $x_{\Lambda} \in X_{q}(\Lambda)$, we must specify some configuration $x_{\mathrm{ext}} \in X_{q}\left(\operatorname{ext} x_{\Lambda}\right)$ such that $x_{\mathrm{ext}}$ has no exterior large contours.

At the same time we must specify some family of arbitrary configurations $x_{W_{\tilde{q}}} \in X_{\widetilde{q}}\left(W_{\tilde{q}}\right), \tilde{q} \in Q$. We get the relation

$$
\begin{aligned}
\mathbf{Z}_{q}(\Lambda, H)= & \sum_{\left\{\Gamma_{\mathrm{cx} t}^{q}\right\}} \mathbf{Z}_{q}^{\mathfrak{Q}}(\operatorname{ext}, H) \prod_{i} \exp \left(-\Phi\left(\Gamma_{\mathrm{ext} i}^{q}\right)\right. \\
& \left.-e_{q}\left|\operatorname{supp} \Gamma_{\mathrm{ext} i}^{q}\right|\right) \prod_{\tilde{q} \in Q} \mathbf{Z}_{\tilde{q}}\left(W_{\tilde{q}}, H\right)
\end{aligned}
$$

Denote by $s_{q}^{\mathfrak{I I}}$ the free energy of the contour model with a contour functional

$$
\begin{array}{ll}
\Psi^{\mathfrak{A}}\left(\Gamma^{q}\right)=\Psi\left(\Gamma^{q}\right), & \text { if } \Gamma^{q} \text { is small, } \\
\Psi^{\mathfrak{I}}\left(\Gamma^{q}\right)=+\infty, & \text { if } \Gamma^{q} \text { is large. }
\end{array}
$$

Obviously, $s_{q}^{\mathfrak{I}}<s_{q}$. Using Proposition 1.6 and the estimate (2.5) we get the bound

$$
\mathbf{Z}_{q}^{\mathfrak{q}}(\operatorname{ext}, H) \leqq \exp \left(C\left|\partial \operatorname{ext}^{c}\right|-h_{q}^{\mathfrak{q}}|\operatorname{ext}|\right)
$$

where $h_{q}^{\mathfrak{2}}=e_{q}-s_{q}^{\mathfrak{2}}$. By the induction assumption for iii),

$$
\mathbf{Z}_{\tilde{q}}\left(W_{\tilde{q}}, H\right) \leqq \exp \left(2 C\left|\partial W_{\tilde{q}}^{c}\right|-h_{0}\left|W_{\tilde{q}}\right|\right)
$$

$\left[C=C_{(2.5)}\right.$ everywhere in the proof of iii)].

Substitute the bounds (1.37), (1.38) into the relation (1.35). We obtain the following estimate: Denoting by $a_{q}^{\mathfrak{l}} \equiv h_{q}^{\mathfrak{A}}-h_{0}$,

$$
\begin{aligned}
\mathbf{Z}_{q}(\Lambda, H) \leqq & \exp \left(-h_{0}|\Lambda|\right) \sum_{\left\{\Gamma_{\text {ext } i\}}^{q}\right.} \exp \left(-a_{q}^{\mathfrak{I}}|\operatorname{ext}|\right) \\
& \cdot \prod_{i} \exp \left(-\Phi\left(\Gamma_{\text {ext }}^{q}\right)+\left(h_{0}-e_{q}\right)\left|\operatorname{supp} \Gamma_{\text {ext } i}^{q}\right|\right) \\
& \cdot \exp \left(C\left(2 \sum_{\tilde{q}}\left|\partial W_{\tilde{q}}^{c}\right|+\left|\partial \operatorname{ext}^{c}\right|\right)\right)
\end{aligned}
$$


Now we use the main lemma of Sect. 2.2. Factorize the contours with respect to the equivalency

$$
\Gamma^{q} \sim \tilde{\Gamma}^{q} \quad \text { iff } \quad \operatorname{supp} \Gamma^{q}=\operatorname{supp} \tilde{\Gamma}^{q} .
$$

For any equivalence class $\left[\Gamma^{q}\right]$, put

$$
\Phi\left(\left[\Gamma^{q}\right]\right)=-\log \sum_{\tilde{\Gamma}^{q} \in\left[\Gamma^{q}\right]} \exp \left(-\Phi\left(\tilde{\Gamma}^{q}\right)\right) .
$$

Notice that by (1.9) there is some universal constant $K$ depending only on $|S|$ such that for each contour $\Gamma^{q}$,

$$
\Phi\left(\left[\Gamma^{q}\right]\right) \geqq\left|\operatorname{supp} \Gamma^{q}\right|(\tau-K) .
$$

Notice also that [see (1.36), (1.37) and the definition of $a_{q}^{\mathfrak{I}}$ ]

$$
a_{q}^{\mathfrak{A}} \geqq a_{q} .
$$

Finally, it is a simple geometric consideration to show that

$$
2 \sum_{\tilde{q} \in Q}\left|\partial W_{\widetilde{q}}^{c}\right|+\left|\partial \operatorname{ext}^{c}\right| \leqq\left|\partial \Lambda^{c}\right|+3 \sum_{i}\left|\operatorname{supp} \Gamma_{\mathrm{ext} i}^{q}\right|
$$

[In fact, it is possible to replace 3 by 2 if dist(ext, $\left.W_{q}\right)>2, q \in Q$.] Factorizing the contours in (1.39) and using the estimates (1.43), (1.41), and (1.42), we get

$$
\begin{aligned}
\mathbf{Z}_{q}(\Lambda, H) \leqq & \exp \left(-h_{0}|\Lambda|+C\left|\partial \Lambda^{c}\right|\right) \\
& \sum_{\left\{\Gamma_{\text {ext }\}}^{q}\right.} \exp \left(-a_{q}^{\mathfrak{2}}|\operatorname{ext}|\right) \prod_{i} \exp \left(-\Phi\left(\left[\Gamma_{\text {ext }}^{q}\right]\right)\right. \\
& \left.+\left(3 C+h_{0}-e_{q}\right)\left|\operatorname{supp} \Gamma_{\text {ext } i}^{q}\right|\right) \\
\leqq & \exp \left(-h_{0}|\Lambda|+C\left|\partial \Lambda^{c}\right|\right) \sum_{\left\{\Gamma_{\text {exti }}^{q}\right\}} \exp (-a|\operatorname{ext}|) \\
& \exp \left(\left(3 C+K-\tau+h_{0}-e_{q}\right) \sum_{i}\left|\operatorname{supp} \Gamma_{\text {ext } i}^{q}\right|\right) .
\end{aligned}
$$

Now, if $\tau$ is sufficiently large (notice that $h_{0}-e_{q} \leqq s_{q} \rightarrow 0$ as $\tau \rightarrow \infty$ ), we can use the estimate (2.13), as explained in detail in Sect. 2.2 [notice that each $\Gamma=\Gamma_{\mathrm{ext} i}^{q}$ satisfies the condition (1.26), and therefore Proposition 2.2 can be applied to verify (2.11)]. We obtain

$$
\left.\mathbf{Z}_{q}(\Lambda, H) \leqq \exp \left(-h_{0}\right)|\Lambda|\right) \exp \left(2 C\left|\partial \Lambda^{c}\right|\right)
$$

where $C=C_{(2.5)}$. But this is the required estimate (1.28).

\subsection{Lipschitz Condition for $s_{q}$}

We will use the following norm on the space of all hamiltonians having a fixed range $r$ :

$$
\|H\|=\sup \left(\left\{\left|e_{q}\right|\right\} \cup\left\{\Phi(\Gamma)|\operatorname{supp} \Gamma|^{-1}\right\}\right) .
$$

Proposition. Let $\left\{H_{\lambda}=H+\lambda \tilde{H}, \lambda \in \mathscr{U}(0) \subset \mathbb{R}\right\},\|\tilde{H}\|=1$ be a family of hamiltonians satisfying the condition (1.9). Denote by $s_{q}(\lambda)$ the free energy of the truncated contour model corresponding to the hamiltonian $H_{\lambda}$ and a given $q \in Q$. Then the onesided 
derivative $\frac{d}{d \lambda( \pm)} s_{q}(\lambda)$ exists and satisfies the inequality

$$
\left|\frac{d}{d \lambda( \pm)} s_{q}(\lambda)\right| \leqq \varepsilon(\tau)
$$

where $\varepsilon(\tau)$ is such that $\lim _{\tau \rightarrow \infty} \varepsilon(\tau)=0$.

Proof. By differentiating $\log \mathbf{Z}_{q}\left(\Lambda, \Psi_{\lambda}^{\prime}\right)$ with respect to $\lambda$ and by taking the limit $\Lambda\urcorner \mathbb{Z}^{v}$, we obtain the formula

$$
\frac{d}{d \lambda( \pm)} S_{q}(\lambda)=\sum_{\Gamma: 0 \in \operatorname{supp} \Gamma} P^{(\lambda)}(\Gamma) \frac{d}{d \lambda( \pm)} \Psi_{\lambda}^{\prime}(\Gamma)
$$

where $P_{\lambda}(\Gamma)$ is the correlation function of $\Gamma$ in the infinite ensemble (with a contour functional $\left.\Psi_{\lambda}^{\prime}\right)$. Use the simple bound

$$
\left|\frac{d}{d \lambda( \pm)} \Psi_{\lambda}^{\prime}(\Gamma)\right| \leqq 2|V(\Gamma)|
$$

[which follows from the inequality $\frac{d}{d \lambda} \log \mathbf{Z}\left(\Gamma, H_{\lambda}\right) \leqq|V(\Gamma)|$ and a similar observation for $\left.\log \mathbf{Z}\left(\Gamma_{\text {red }}, H_{\lambda}\right)\right]$. Use also the Peierls inequality

$$
P_{\lambda}(\Gamma) \leqq \exp \left(-\Psi_{\lambda}^{\prime}(\Gamma)\right)
$$

By substituting (1.49), (1.50) into (1.48), we get

$$
\left|\frac{d}{d \lambda( \pm)} s_{q}(\lambda)\right| \leqq 2 \sum_{\Gamma: \operatorname{supp} \Gamma \ni 0} \exp \left(-\frac{\tau}{3}|\operatorname{supp} \Gamma|\right)|V(\Gamma)| \rightarrow 0,
$$

as $\tau \rightarrow \infty$. q.e.d.

\subsection{Investigation of the Phase Diagram}

Theorem. Let $v \geqq 2$, let $S$ be a finite set. Let $H_{0}$ be some finite range hamiltonian invariant with respect to all shifts on $S^{\mathbb{Z}^{v}}$. Let $H_{1}, \ldots, H_{m-1}$ be another hamiltonians of the same type. Consider a family

$$
\left\{H_{\lambda}=H_{0}+\sum_{i=1}^{n-1} \lambda_{i} H_{i}, \lambda=\left(\lambda_{1}, \ldots, \lambda_{m-1}\right) \in \mathscr{U}\right\}
$$

$\mathscr{U}$ being some neighborhood of zero in $\mathbb{R}^{n-1}$. Suppose that there is a fixed family $\left\{x_{q}, q \in Q\right\}, Q \subset S,|Q|=n$ of constant configurations, such that the following conditions are satisfied:

i) all $e_{q}\left(H_{0}\right), q \in Q$ are the same,

ii) let $q_{1}, q_{2}, \ldots, q_{n}$ be same ordering of $Q$. Then the vectors $\mathbf{e}_{i}$ $\equiv\left(e_{q_{1}}\left(H_{i}\right), \ldots, e_{q_{n}}\left(H_{i}\right)\right)$ are independent and their linear span $\mathbf{L}$ does not contain the vector $(1, \ldots, 1)$ ( degeneracy removing assumption),

iii) the condition (1.9) is satisfied for all $H=H_{\lambda}, \lambda \in \mathscr{U}$ with a sufficiently large $\tau$ (therefore, $\left\{x_{q}, q \in Q\right\}$ is just the set of all ground states - in the usual sense of PS theory). 
Given $\mathbf{h} \in \mathbb{R}^{n}$, denote by

$$
\mathbf{h}^{0}=\mathbf{h}-\left(\min _{k=1, \ldots, n}\left\{\mathbf{h}_{k}\right\}\right)(1, \ldots, 1) .
$$

Denote also by $\left(\mathbb{R}^{n}\right)^{0}=\left\{\mathbf{h}^{0}, \mathbf{h} \in \mathbb{R}^{n}\right\}$. Then, for some neighborhood of zero $\mathscr{V} \subset\left(\mathbb{R}^{n}\right)^{0}$, there is a homeomorphic mapping

$$
\left\{\mathbf{h}^{0} \leadsto \lambda\left(\mathbf{h}^{0}\right)\right\},
$$

defined on $\mathscr{V}$ and such that for each $\mathbf{h}^{0} \in \mathscr{V}$,

$$
\mathbf{h}^{0}\left(\lambda\left(\mathbf{h}^{0}\right)\right)=\mathbf{h}^{0},
$$

where $\mathbf{h}(\lambda)$ denotes the vector $\left(h_{q_{1}}(\lambda), h_{q_{2}}(\lambda), \ldots, h_{q_{n}}(\lambda)\right)$. In particular, $q \in Q$ is stable with respect to the hamiltonian

$$
H_{\lambda}, \lambda=\lambda\left(\mathbf{h}^{0}\right) \quad \text { iff } \quad \mathbf{h}_{q}^{0}=0 .
$$

Notes. i) The precise bound for $\tau$ will be specified later. It depends on $\mathscr{U}$ and $\left\{\mathbf{e}_{i}\right\}$.

ii) Denote by $Q(\lambda)=\left\{q \in Q: q\right.$ is stable with respect to $\left.H_{\lambda}\right\}$. For any $\lambda \in \mathscr{U}$ and any $q \in Q(\lambda)$ we can construct, using the appropriate contour model [given by $\left.\Psi_{\lambda}\left(\Gamma^{q}\right)\right]$ and Proposition 1.6, some Gibbs state $P_{\lambda}^{q}$ on $S^{\mathbb{Z}^{v}}$. We will not give the details of this construction (see [2]). The mapping (1.53) is usually called the phase diagram. We will not investigate further properties (analyticity, for example) of the manifolds

$$
\{\lambda \in \mathscr{U}: Q(\lambda)=\bar{Q}\} \quad \bar{Q} \subset Q,
$$

respectively

$$
\left\{\left(\lambda, \mathbf{h}_{q}(\lambda)\right): \bar{Q}(\lambda)=\bar{Q}\right\} \quad(q \in \bar{Q}) .
$$

This will be the subject of a forthcoming paper.

Proof of Theorem. Denote by symbol $\mathscr{L}$ the linear mapping

$$
\left\{\lambda \leadsto \sum_{i=1}^{n-1} \lambda_{i} \mathbf{e}_{i}\right\}: \mathbb{R}^{n-1} \rightarrow \mathbf{L} .
$$

Given $\mathbf{h} \in \mathbb{R}^{n}$, denote by $[\mathbf{h}]$ the ray $\left\{\mathbf{h}^{\prime} \in \mathbb{R}^{n}: \mathbf{h}^{\prime 0}=\mathbf{h}^{0}\right\}$, and denote by $\mathbf{h}^{\mathbf{L}}$ the intersection of $[\mathbf{h}]$ and $\mathbf{L}$. Consider, on $\mathscr{U}$, the mapping

$$
\mathscr{F}(\lambda)=\lambda-\mathscr{L}^{-1}\left(\mathbf{h}^{\mathbf{L}}(\lambda)\right)
$$

and also the mappings

$$
\mathscr{F}_{\mathbf{h}}(\lambda)=\lambda-\mathscr{L}^{-1}\left(\mathbf{h}^{\mathbf{L}}(\lambda)-\mathbf{h}^{\mathbf{L}}\right)
$$

(the values of $\mathbf{h}$ being specified later).

We want to solve the equation

$$
\mathscr{F}_{\mathbf{h}}(\lambda)=\lambda .
$$

Let us show that $\mathscr{F}$ (and therefore $\mathscr{F}_{\mathbf{h}}$, too) is a contraction mapping on $\mathscr{U}$. Because

$$
\mathscr{F}(\lambda)-\mathscr{F}\left(\lambda^{\prime}\right)=\mathscr{L}^{-1}\left(\mathbf{s}^{\mathbf{L}}(\lambda)-\mathbf{s}^{\mathbf{L}}\left(\lambda^{\prime}\right)\right),
$$


[where $\mathbf{s}(\lambda)=\left(s_{q_{1}}(\lambda), s_{q_{2}}(\lambda), \ldots, s_{q_{n}}(\lambda)\right]$, and because

$$
\left\|\mathscr{L}^{-1}\left(\mathbf{s}^{\mathbf{L}}(\lambda)-\mathbf{s}^{\mathbf{L}}(\lambda)\right)\right\| \leqq\left|\lambda-\lambda^{\prime}\right|\left\|\mathscr{L}^{-1}\right\| \varepsilon(\tau)(\sin \alpha)^{-1} n^{1 / 2},
$$

where $|\lambda|^{2}=\sum_{i}\left|\lambda_{i}\right|^{2},|\mathbf{h}|^{2}=\sum_{q}\left|h_{q}\right|^{2}$, accordingly is defined $\left\|\mathscr{L}^{-1}\right\|, \varepsilon(\tau)$ was defined in (1.47) and $\alpha$ is the angle between $\mathbf{L}$ and $(1, \ldots, 1)$, we see that $\mathscr{F}$ is a contraction if $\tau$ is sufficiently large. Denote by $\kappa$ the contraction constant of $\mathscr{F}$. Because

$$
\|\mathscr{F}(0)\|=\| \mathscr{L}^{-1}\left(-s^{\mathbf{L}}(0) \| \leqq \varepsilon^{\prime}(\tau) \rightarrow 0,\right.
$$

as $\tau \rightarrow \infty$, we may further assume that there is a neighborhood of zero $\mathscr{V} \subset\left(\mathbb{R}^{n}\right)^{0}$ and a ball $B_{r}=\{\lambda:\|\lambda\| \leqq r\} \subset \mathbb{R}^{n-1}$ such that $\mathscr{F}_{\mathbf{h}}(0)=\mathscr{L}^{-1}(\mathbf{h}-\mathbf{s}(0)) \in B_{r}$ for each $\mathbf{h} \in \mathscr{V}$ and also

$$
B_{r} \subset(1-\kappa) \mathscr{U}
$$

Put

$$
\lambda(\mathbf{h})=\lim _{n \rightarrow \infty}\left(\mathscr{F}_{\mathbf{h}}\right)^{n}(0), \quad \mathbf{h} \in \mathscr{V} .
$$

By (1.64), $\lambda(\mathbf{h}) \in \mathscr{U}$. Because $\mathbf{h}^{\mathbf{L}}(\lambda)=\mathbf{h}$ for $\lambda=\lambda(\mathbf{h})$, we see that (1.54) is satisfied. Clearly, $\{\mathbf{h} \rightarrow \lambda(\mathbf{h})\}$ is the desired homeomorphism. q.e.d.

\section{Main Lemma}

In this section, the technical tools used in both Sects. 1 and 3 are collected. First, in Sect. 2.1, we present some rudiments of the theory of "polymer" models and the method of the cluster expansion, without proof. In Sect. 2.2 we formulate the main lemma. It describes, in the simplest model situation, the behaviour of the system with "unstable" boundary condition, especially the formation of large contours with a "stable" phase in its interior. Finally, in Sect. 2.3 we generalize the main lemma in view of its applications in Sect. 3.

\subsection{Polymer Models}

Suppose that some real or complex function $k_{T}$ of finite connected subsets $T$ of $\mathbb{Z}^{v}$ is given. We define, for each finite $\Lambda \subset \mathbb{Z}^{v}$ the "polymer" partition function

$$
\mathbf{Z}_{\Lambda}=\sum_{\left\{T_{i}\right\}} \prod_{i} k_{T_{i}}
$$

the sum being taken over all families $\left\{T_{i}\right\}$ of connected sets such that $\operatorname{dist}\left(T_{i}, T_{i^{\prime}}\right) \geqq 2$ for any $i \neq i^{\prime}$ and $\operatorname{dist}\left(V\left(T_{i}\right), \Lambda^{c}\right) \geqq 2$ for any $i$ (see 2.2).

Proposition. Suppose that the functions $k_{T}$ are invariant with respect to all shifts in $\mathbb{Z}^{v}$. Suppose moreover that the condition

$$
\left|k_{T}\right| \leqq \exp (-\tau|T|)
$$

is satisfied for each $T$, with a sufficiently large $\tau$. Then $\mathbf{Z}_{\Lambda} \neq 0$ for any finite $\Lambda \subset \mathbb{Z}^{\nu}$. The limit

$$
S=\lim _{\Lambda \ngtr \mathbb{Z}^{v}}|\Lambda|^{-1} \log \mathbf{Z}_{\Lambda}
$$


exists, with a proper choice of $\operatorname{Im} \log \mathbf{Z}_{\Lambda}$, as an analytical function of all the variables $k_{T}$, and satisfies the estimate

$$
S<\sum_{T: T \ni 0}\left|k_{T}\right|
$$

In particular, $s \rightarrow 0$ as $\tau \rightarrow \infty$. Finally there is an universal constant $C=C(\tau)$ such that $C \rightarrow 0$ as $\tau \rightarrow \infty$ and for each finite $\Lambda \subset \mathbb{Z}^{v}$,

$$
\left|\log \mathbf{Z}_{\Lambda}-s\right| \Lambda||<C\left|\partial \Lambda^{c}\right|
$$

where $\partial \Lambda^{c}=\left\{t \in \Lambda: \operatorname{dist}\left(t, \Lambda^{c}\right)=1\right.$.

Usually, we will use (2.5) for the contour models: if $\Psi\left(\Gamma^{q}\right)$ is a contour functional, we can define its factorization

$$
k_{T}=\sum_{\Gamma^{q}: \operatorname{supp} \Gamma^{q}=T} \exp \left(-\Psi\left(\Gamma^{q}\right)\right) .
$$

Obviously, $\mathbf{Z}_{q}(\Lambda, \Psi)=\mathbf{Z}_{\Lambda}$ in such a case and we can use the expression (2.5) for $\mathbf{Z}_{\Lambda}$.

ii) We omit the proof of the proposition. The only nonelementary statement is (2.5), which is proven using the method of the cluster expansion. Such statements were used by many authors (also in [1], but see e.g. [5,6] for a more recent references). They are based on the expression of the correlation functions by cluster expansion series.

\subsection{Main Lemma}

Consider the following artificial model.

Definition 1. By a contour everywhere in Sect. 2.2 we mean, a finite connected subset of $\mathbb{Z}^{v}$. Given any contour $T$, we denote by int $T$ the union of all inner components of $T^{c}$. We denote by $V(T)=T \cup \operatorname{int} T$.

Definition 2. Fix some finite volume $\Lambda \subset \mathbb{Z}^{v}$. By a configuration in $\Lambda$ we mean a consistent choice of external contours $\left\{T_{i}\right\}$ in $\Lambda$ everywhere in Sect. 2.2, i.e. such a choice of contours $\left\{T_{i}\right\}$ that $V\left(T_{i}\right) \subset \Lambda \backslash \partial \Lambda$ for each $i$ and $\operatorname{dist}\left(V\left(T_{i}\right), V\left(T_{i^{\prime}}\right)\right) \geqq 2$ whenever $i \neq i^{\prime}$. Denote by $X_{\text {ext }}(\Lambda)$ the set of all such configurations. Given any configuration $x_{\Lambda} \equiv\left\{T_{i}\right\} \in X_{\text {ext }}(\Lambda)$, denote by

$$
\operatorname{ext} x_{\Lambda} \equiv \operatorname{ext}\left\{T_{i}\right\}=\Lambda \bigcup_{i} \operatorname{int} T_{i}
$$

[a slight difference from (1.34)].

Definition 3. Suppose that some constant $a>0$ and some function $\Phi(T)$ ("contour hamiltonian") is given. Define a hamiltonian of any configuration $x_{\Lambda} \in X_{\text {ext }}(\Lambda)$ as

$$
H\left(x_{\Lambda}\right)=a\left|\operatorname{ext} x_{\Lambda}\right|+\sum_{i} \Phi\left(T_{i}\right) .
$$

Assumption. Suppose that there is a sufficiently large $\tau>0$ such that for each contour $T$,

$$
\Phi(T)>\tau|T| .
$$


Suppose also that $\Phi(T)$ are invariant with respect to all shifts.

Definition 4. Let $C$ be the constant from (2.5). [We will assume that $\tau, C$ are such that $C \geqq C\left(\tau^{\prime}\right)$, where $\tau^{\prime}=\tau-C$.] Define an auxiliary contour functional

$$
\Phi^{*}(T)=\Phi(T)-C|T| .
$$

Definition 5. Denote by $s^{*}$ the free energy of the polymer model with a weight $k_{T}=\exp \left(-\Phi^{*}(T)\right)$.

Main Lemma. Consider the situation described so far in Sect. 2.2. Assume that

$$
s^{*}<a .
$$

Denote by

$$
\mathbf{Z}(\Lambda, H)=\sum_{x_{\Lambda} \in X_{\mathrm{ext}}(\Lambda)} \exp \left(-H\left(x_{\Lambda}\right)\right),
$$

the hamiltonian $H\left(x_{A}\right)$ being given by (2.8). Then

$$
\mathbf{Z}(\Lambda, H)<\exp C\left|\partial \Lambda^{c}\right| .
$$

Notes. i) (2.11) is satisfied e.g. in the case when

$$
\sum_{T: T \ni 0} \exp \left(-\Phi^{*}(T)\right)<a
$$

[compare (2.4)]. A simple example when (2.14) is valid will be shown later in the proposition. This example will also explain our use of the main lemma in the proof of Theorem 1 of Sect. 1.

ii) To obtain some interpretation of the main lemma (and to indicate its use in Sect. 3) replace (2.12) by a stronger inequality

$$
s^{*}<\frac{1}{2} a \text {. }
$$

Say that a point $t \in \Lambda$ is an unstable point of a configuration

$$
x_{\Lambda}=\left\{T_{i}\right\} \in X_{\text {ext }}(\Lambda) \text { if } t \in \operatorname{ext} x_{\Lambda} .
$$

Denote by $\mathbf{Z}_{N}(\Lambda, H)$ the partition function corresponding to configurations from $X_{\text {ext }}(\Lambda)$ with at least $N$ unstable points. Then (2.13) gives the estimate [notice that (2.11) is replaced by (2.15)]

$$
\mathbf{Z}_{N}(\Lambda, H)<\exp \left(-\frac{a}{2} N\right) \exp \left(C\left|\partial \Lambda^{c}\right|\right) .
$$

Suppose, on the other hand, that the condition

$$
\mathbf{Z}(\Lambda, H)>\exp \left(-C^{\prime}\left|\partial \Lambda^{c}\right|\right)
$$

is satisfied, with another constant $C^{\prime}$ (such a condition will be satisfied in any case which we will be interested in). Then, a typical configuration from $X_{\text {ext }}(\Lambda)$ has only $O\left|\partial \Lambda^{c}\right|$ unstable points.

Proof of the Main Lemma. Consider the auxiliary polymer model with a weight

$$
k_{T}=\exp \left(-\Phi^{*}(T)\right) \text {. }
$$


Use the notation $\mathbf{Z}_{\Lambda}$ for the partition function of this polymer model. By (2.5),

$$
\mathbf{Z}_{\text {int } T} \exp \left(-S^{*}|\operatorname{int} T|\right) \exp (-C|T|)<1
$$

for any contour $T$. Thus, if we "fill in" each set int $T$ by other contours we get, using (2.18), the inequality

$$
\begin{aligned}
\mathbf{Z}(\Lambda, H) & =\sum_{X_{\mathrm{ext}}(\Lambda)} \exp \left(-a\left|\operatorname{ext} x_{\Lambda}\right|\right) \prod_{i} \exp \left(-\Phi\left(T_{i}\right)\right) \\
& \leqq \sum_{X_{\mathrm{ext}}(\Lambda)} \exp \left(-a\left|\operatorname{ext} x_{\Lambda}\right|\right) \prod_{i} \exp \left(-\Phi^{*}\left(T_{i}\right)-s^{*}\left|\operatorname{int} T_{i}\right| \mathbf{Z}_{\mathrm{int} T_{\imath}}\right. \\
& \leqq \exp \left(-s^{*}|\Lambda|\right) \mathbf{Z}_{\Lambda} \leqq \exp \left(C\left|\partial \Lambda^{c}\right|\right) . \quad \text { q.e.d. }
\end{aligned}
$$

Definition 6. Say that $T$ is a stable contour if

$$
\tau|T|>a|V(T)| .
$$

Say that $T$ is a large contour if there is an unstable contour $\tilde{T}$ such that

$$
\tilde{T} \subset \operatorname{int} T
$$

[compare the analogous notions of Sect. 1 and (1.26)].

Proposition. Suppose that (2.9) is valid and moreover suppose that $\Phi^{*}(T)=+\infty$ for all small contours $T$. Then (2.14) is valid.

Proof. As a first step we will prove:

Lemma 1. There are universal constants $K>0$ and $\kappa>0$ such that any large contour satisfies the inequality

$$
|T| \geqq K\left(\frac{\tau}{a}\right)^{\kappa} .
$$

Proof of Lemma 1. Because

$$
V(T)=O\left((\operatorname{diam} T)^{v}\right)
$$

and

$$
\operatorname{diam} T \leqq|T|
$$

we see from (2.20) that unstable contours satisfy the inequality

$$
\operatorname{diam} T \geqq K\left(\frac{\tau}{a}\right)^{\frac{1}{v-1}}
$$

for some universal constant $K>0$. By (2.21) we obtain (2.25) also for any large contour. Using (2.24) we obtain (2.22). q.e.d.

Note. More accurate estimates of $\kappa, K$ can be obtained using the isoperimetric inequality.

Now we can prove (2.14). We use the estimate $\tilde{K}^{N}$ [where $\tilde{K}(=\tilde{K}(v))$ is an universal constant] for the number of contours $T$ such that $T \ni 0$ and $|T|=N$. We use also the estimate (2.9) and definition (2.10), with $\tau$ sufficiently large. Obviously.

$$
\begin{aligned}
& \sum_{T \text { large }: T \ni 0} \exp \left(-\Phi^{*}(T)\right) \leqq \\
&=\frac{1}{N \geqq K\left(\frac{\tau}{a}\right)^{\kappa}} \exp ((C-\tau) N) \tilde{K}^{N} \\
&=\frac{\exp C^{\prime}}{1-} \exp \left(C^{\prime} K\left(\frac{\tau}{a}\right)^{\kappa}\right), \quad C^{\prime}=C-\tau+\log \tilde{K} .
\end{aligned}
$$


The last expression of (2.26) is smaller than $a$, for a sufficiently large $\tau$, because of the trivial relation $q^{\left(\frac{\tau}{a}\right)^{\kappa}}=o(a), a \rightarrow 0, q<1$.

\subsection{Generalization of Main Lemma}

Consider the following model.

Definition 1. By a contour everywhere in Sect. 2.3 we mean a pair

$$
\Gamma^{q}=\left(\operatorname{supp} \Gamma^{q}, \operatorname{sign}_{\Gamma^{q}}\right),
$$

where $\operatorname{supp} \Gamma^{q}$ is a connected set and $\operatorname{sign}_{\Gamma^{q}}(\cdot)$ is a function of components of $\left(\operatorname{supp} \Gamma^{q}\right)^{c}$ having values from $\{0,1,2, \ldots, n\}$, the exterior component of $\left(\operatorname{supp} \Gamma^{q}\right)^{c}$ being signed by a nonzero value $q$.

We denote by $V(\Gamma)=V(\operatorname{supp} \Gamma)($ see Sect. 2.2, Definition 1$)$. Also, we denote by int $_{q} \Gamma$ the union of all interior $q$-components of the set $(\operatorname{supp} \Gamma)^{c}$. We consider the pair $(\phi, q)$ as a contour.

Definition 2. By a configuration $x_{A}$ in a finite $\Lambda \subset \mathbb{Z}^{v}$ everywhere in Sect. 2.3 we mean a consistent choice of contours $\left\{\Gamma_{i}\right\}$ containing $(\phi, q)$ for some nonzero $q$, consistency being meant in the sense that: i) $V\left(\Gamma_{i}\right) \subset \backslash \partial \Lambda$ for each $i$,

ii) $\operatorname{dist}\left(\Gamma_{i}, \Gamma_{i^{\prime}}\right) \geqq 2, i \neq i^{\prime}$ and if $C$ is a component of $\Lambda \backslash \bigcup_{i} \operatorname{supp} \Gamma_{i}$ and $\left\{\tilde{\Gamma}_{k}\right\} \subset\left\{\Gamma_{i}\right\}$ is the set of all contours which touch $C$, including $(\phi, q)$ if $C \supset \Lambda^{c}$, then the functions $\operatorname{sign}_{\tilde{\Gamma}_{k}}(\cdot)$ coincide on $C$,

iii) $\operatorname{int}_{0} \Gamma_{i} \cap\left(\bigcup_{i^{\prime}: i^{\prime} \neq i} \operatorname{supp} \Gamma_{i^{\prime}}\right)=\emptyset$ for each $i$.

Definition 3. Suppose that some $a_{q}>0$ and also some "contour hamiltonians" $\Phi\left(\Gamma^{q}\right)$ are given for $q=1, \ldots, n$. Denote by $\Lambda_{q}\left\{\Gamma_{i}\right\}$ the set of all $t \in \Lambda$ which are either $q$-correct (i.e. are signed $q$ by any of the function $\operatorname{sign}_{\tilde{\Gamma}_{k}}$ mentioned in Definition 2, ii)) or belong to the support of some $q$-contour from the system $\left\{\Gamma_{i}\right\}$. Define the hamiltonian

$$
H\left(x_{\Lambda}\right)=\sum_{q=1}^{n} a_{q}\left|\Lambda_{q}\left(x_{\Lambda}\right)\right|+\sum_{i} \Phi\left(\Gamma_{i}\right), \quad x_{\Lambda} \equiv\left\{\Gamma_{i}\right\} .
$$

Suppose that all $\Phi(\Gamma)$ are invariant with respect to shifts in $\mathbb{Z}^{v}$. Suppose that a Peierls condition

$$
\Phi(\Gamma)>\tau|\operatorname{supp} \Gamma|
$$

is satisfied for each $\Gamma$, with a sufficiently large $\tau$. Define the auxiliary contour functionals

$$
\Phi^{*}(\Gamma)=\Phi(\Gamma)-C|\operatorname{supp} \Gamma|
$$

where $C$ is from (2.5). Define the classes of equivalency $\left[q, \operatorname{supp} \Gamma^{q}\right]$ consisting of all $\Gamma^{q}$ with the same $q$ and $\operatorname{supp} \Gamma^{q}$, and define the factorized contour functionals

$$
\Phi\left(\left[q, \operatorname{supp} \Gamma^{q}\right]=-\log \sum_{\tilde{\Gamma}^{q} \in\left[q, \operatorname{supp} \Gamma^{q}\right]} \exp \left(-\Phi\left(\tilde{\Gamma}^{q}\right) .\right.\right.
$$


Notice that (2.28) implies the inequality

$$
\Phi\left(\left[q, \operatorname{supp} \Gamma^{q}\right]\right) \geqq(\tau-L)\left|\operatorname{supp} \Gamma^{q}\right|
$$

for some universal constant $L$ depending only on $n$. Denote by $s_{q}^{*}$ the free energy of the polymer model with a weight

$$
k_{\mathrm{supp} \Gamma}=\exp \left(-\Phi^{*}\left[q, \operatorname{supp} \Gamma^{q}\right]\right) .
$$

Assume that for each $q=1, \ldots, n$ the inequality

$$
s_{q}^{*} \leqq a_{q}
$$

is satisfied. Assume that $\tau, C$ are such that $C \geqq C\left(\tau^{\prime}\right)$, where

$$
\tau^{\prime}=\tau-L-2 C \text {. }
$$

For any finite $\Lambda \subset \mathbb{Z}^{v}$ and any $q$, denote by $X_{q}(\Lambda)$ the set of all configurations (in the sense of this Sect. 2.3) $x_{A} \equiv\left\{\Gamma_{i}\right\}$ on $\Lambda$ such that $\left\{\Gamma_{i}\right\} \ni(\phi, q)$, i.e. the exterior contours of $x_{A}$ are $q$-contours. Put

$$
\mathbf{Z}_{q}(\Lambda, H)=\sum_{X_{q}(\Lambda)} \exp \left(-H\left(x_{\Lambda}\right)\right)
$$

Then

$$
\mathbf{Z}_{q}(\Lambda, H) \leqq \exp \left(C\left|\partial \Lambda^{c}\right|\right)
$$

Proof. Introduce again the notions of a level of a contour and of a level of a finite set $\Lambda \subset \mathbb{Z}^{v}$ (see Sect. 1, Proposition 1.7). We will prove (2.35) by induction on the level of $\Lambda$. First notice that the case when $\Lambda$ has a level 0 is trivial. To prove the induction step we will use the main lemma. Given any configuration $x_{\Lambda} \equiv\left\{\Gamma_{i}\right\}$ (in the sense of this section) denote by $\left\{\Gamma_{\mathrm{ext} j}^{q}\right\}$ its external contour system. Fix $\left\{\Gamma_{\mathrm{ext} j}^{q}\right\}$ and notice that each set $\Lambda_{j}^{\tilde{q}} \equiv \operatorname{int}_{\tilde{q}}\left(\Gamma_{\mathrm{ext} j}^{\tilde{q}}\right)$ has a level smaller than the level of $\Lambda$. We can therefore use the induction assumption for $\mathbf{Z}_{\tilde{q}}\left(\Lambda_{j}^{\tilde{q}}, H\right)$. Clearly, if we denote by $\operatorname{ext}\left(=\operatorname{ext} x_{\Lambda}\right)$ the set $A \bigcup_{j}$ int $\Gamma_{\text {ext } j}^{q}$, then

$$
\begin{aligned}
\mathbf{Z}_{q}(\Lambda, H)= & \sum_{\left\{\Gamma_{\text {ext }\}}\right\}} \exp \left(-a_{q}\left|\operatorname{ext} x_{\Lambda}\right|\right) \prod_{j} \exp \left(-\Phi\left(\Gamma_{\text {ext } j}^{q}\right)\right) \prod_{\tilde{q}} \mathbf{Z}_{\tilde{q}}\left(\Lambda_{j}^{\tilde{q}}, H\right) \\
\leqq & \sum_{\left\{\left[q, \operatorname{supp} \Gamma_{\text {ext } j}^{q}\right]\right\}}\left(-a_{q}|\operatorname{ext}| \prod_{j} \exp \left(-\Phi\left(\left[q, \operatorname{supp} \Gamma_{\text {ext } j}^{q}\right]\right)\right.\right. \\
& \left.+C^{\prime}\left|\operatorname{supp} \Gamma_{\text {ext } j}^{\tilde{q}}\right|\right),
\end{aligned}
$$

where $\Phi$ is the factorized functional [see (2.30)] and where we used the inequality [compare (1.43)]

$$
\sum_{j} \sum_{\tilde{q}}\left|\partial\left(\Lambda_{j}^{\tilde{q}}\right)^{c}\right| \leqq \sum_{j}\left|\operatorname{supp} \Gamma_{\mathrm{ext} i}^{q}\right|
$$

Now we use (2.31). By (2.34), $\tau-2 C-L$ is sufficiently large such that (2.13) could be applied to the last sum in (2.36): we obtain

$$
\begin{aligned}
\mathbf{Z}_{q}(\Lambda, H) & \leqq \sum_{\left\{\left[q, \operatorname{supp} \Gamma_{\text {ext j }]\}}^{q}\right.\right.} \exp \left(-a_{q}|\operatorname{ext}|\right) \prod_{j} \exp \left(-\Phi^{*}\left(\left[q, \operatorname{supp} \Gamma_{\operatorname{ext} j}^{q}\right]\right)\right. \\
& \leqq \exp \left(C\left|\partial \Lambda^{c}\right|\right) . \quad \text { q.e.d. }
\end{aligned}
$$




\section{Completeness of the Diagram}

\subsection{Basic Ideas}

We consider again the model introduced in Sect. 1 (1.1-1.5).

Definition 1. Given any configuration $x_{\Lambda} \in X_{q}(\Lambda)$ [see (1.10)], denote by $\mathscr{L}$ $\left(=\mathscr{L}\left(x_{A}\right)\right)$ the system of all large contours $\Gamma^{q}$ of $x_{A}$ which satisfy the following condition:

$$
\operatorname{supp} \Gamma^{q} \mathrm{C} \operatorname{int} \tilde{\Gamma}^{\tilde{q}} \text { for no small contour } \tilde{\Gamma}^{\tilde{q}} \text { of } x_{\Lambda} \text {. }
$$

Definition 2. Given $x_{\Lambda} \in X_{q}(\Lambda)$ consider a canonical configuration $x_{\Lambda}^{\mathscr{L}}$ corresponding to the configuration $x_{A}$, defined as follows:

i) $\left(x_{\Lambda}^{\mathscr{L}}\right)_{t}=\left(x_{A}\right)_{t}$ for $t \in \bigcup_{\Gamma \in \mathscr{L}} \operatorname{supp} \Gamma$.

ii) If $C$ is a component of the set $\Lambda \bigcup_{\Gamma \in \mathscr{L}} \operatorname{supp} \Gamma$, then $x_{\Lambda}^{\mathscr{L}}$ has a constant value on $C \cup \partial C^{c}$.

Definition 3. A stable domain of $x_{\Lambda} \in X_{q}(\Lambda)$ is defined as the union of all components of $\Lambda \bigcup_{\Gamma \in \mathscr{L}} \operatorname{supp} \Gamma$ which have a stable value (in the sense of Definition 5 of 1.7) in the canonical configuration $x_{A}^{\mathscr{L}}$. The points of stable domains will be called stable points of the configuration $x_{\Lambda} \in X_{q}(\Lambda)$.

Note. If $t \in \Lambda$ is a stable point of $x_{\Lambda} \in X_{q}(\Lambda)$, then either $q$ is stable or the following condition is satisfied: there is a contour $\Gamma$ of $x_{A}$ and a stable $\tilde{q}$ such that $t \in \operatorname{int}_{\tilde{q}} \Gamma$. The converse statement is not true because for a nonstable $q$ there may be a small contour $\Gamma^{q}$ such that $\operatorname{int}_{\tilde{q}} \Gamma^{q} \neq \emptyset$ for some stable $\tilde{q}$.

Proposition. Denote by $X_{q}^{N}(\Lambda)$ the subset of $X_{q}(\Lambda)$ consisting of configurations with at least $N$ unstable points. Denote by

$$
\mathbf{Z}_{q}^{N}(\Lambda, H)=\sum_{x_{\Lambda} \in X_{q}^{N}(\Lambda)} \exp \left(-H\left(x_{\Lambda} \mid\left(x_{q}\right)_{\Lambda^{c}}\right) .\right.
$$

Let $0<\alpha<1$. Let $\tau$ in (1.9) be sufficiently (depending on $\alpha$ ) large. Then

$$
\mathbf{Z}_{q}^{N}(\Lambda, H)<\exp \left(-h_{0}|\Lambda|-\alpha a N+C\left|\partial \Lambda^{c}\right|\right)
$$

with some constant $C=C(\tau)$, such that $C \rightarrow 0$ as $\tau \rightarrow \infty$.

Note. This statement is an essential step in the proof of the forthcoming Theorem 3.2. In the proof of (3.5), we use the results of Sect. 2, Lemma 2.3 and Proposition 2.2 in particular.

Proof. The summation over $X_{q}^{N}(\Lambda)$ in (3.4) will be carried out in two steps: 1) Fix some canonical configuration $x_{\Lambda}^{\mathscr{E}}, x_{\Lambda} \in X_{q}^{N}(\Lambda)$, and sum over all $x_{\Lambda} \in X_{q}(\Lambda)$ with the same $x_{\Lambda}^{\mathscr{L}}$. Let $\mathscr{L}=\left\{\Gamma_{i}\right\}$. Denote by $W_{1}, \ldots, W_{m}$ the components of $\Lambda \bigcup_{i} \operatorname{supp} \Gamma_{i}$. Notice that each $x_{A}$ from the equivalence class given by $x_{A}^{\mathscr{L}}$ satisfies the following property: its restrictions to any $W_{j}$ have only small external contours. Actually, this is, assuming that all $\Gamma_{i}$ are contours of $x_{A}$, an equivalent characterization of those $x_{\Lambda} \in X_{q}(\Lambda)$, which give the same $x_{\Lambda}^{\mathscr{L}}$. 
2) Now debote by $\operatorname{CAN}_{q}^{N}(\Lambda)$ the set of all possible $\mathscr{L}\left(x_{\Lambda}\right), x_{\Lambda} \in X_{q}^{N}(\Lambda)$, and sum over all $\mathscr{L} \in \operatorname{CAN}_{q}^{N}(\Lambda)$ : writing

$$
\Phi^{e}\left(\Gamma^{q}\right)=\Phi\left(\Gamma^{q}\right)+e_{q}\left|\operatorname{supp} \Gamma^{q}\right|
$$

we get the relation

$$
\mathbf{Z}_{q}^{N}(\Lambda, H)=\sum_{\mathscr{L} \in \mathrm{CAN}_{q}^{N}(\Lambda)} \prod_{i} \exp \left(-\Phi^{e}\left(\Gamma_{i}\right)\right) \prod_{j} \mathbf{Z}_{q\left(W_{j}\right)}^{\mathfrak{I}}\left(W_{j}, H\right)
$$

where $\mathbf{Z}_{q}^{\mathfrak{Q}}(W, H)$ denotes the partition function corresponding to the configurations which have only small external contours (the same notation as in the proof of iii), Theorem 1.6) and $q_{\left(W_{j}\right)}$ is the value of $x_{\Lambda}^{\mathscr{L}}$ in $W_{j}$. Using the very definition of a small contour and using Proposition 1.6, we can rewrite (3.6) as

$$
\sum_{\mathscr{L} \in \mathrm{CAN}_{q}^{N}(\Lambda)} \prod_{i} \exp \left(-\Phi^{e}\left(\Gamma_{i}\right)\right) \prod_{j} \exp \left(-e_{q\left(W_{j}\right)}\left|W_{j}\right|\right) Z_{q\left(W_{j}\right)}^{\mathfrak{A}}\left(W_{j}, \Psi^{\prime}\right) .
$$

Using (2.5) for the factorized weight

$$
k_{T}^{q}=\sum_{\Gamma^{q}: \operatorname{supp} \Gamma^{q}=T} \exp \left(-\Psi^{\prime}\left(\Gamma^{q}\right)\right)
$$

we get the inequality

$$
\mathbf{Z}_{q}^{\mathfrak{Q I}}\left(W, \Psi^{\prime}\right) \leqq \mathbf{Z}_{q}\left(W, \Psi^{\prime}\right) \leqq \exp \left(s_{q}|W|+C\left|\partial W^{c}\right|\right)
$$

with the same constant $C$ as in (2.5). Substituting this into (3.7) and (3.6), we get

where

$$
\begin{aligned}
\mathbf{Z}_{q}^{N}(\Lambda, H) \leqq & \sum_{\mathscr{L} \in \operatorname{CAN}_{q}^{N}(\Lambda)} \exp \left(-\sum_{i} \Phi^{e}\left(\Gamma_{i}\right)\right) \prod_{j} \exp \left(-h_{q\left(W_{J}\right)}\left|W_{j}\right|+C\left|\partial W_{j}^{c}\right|\right) \\
\leqq & \exp \left(-h_{0}|\Lambda|\right) \exp \left(C\left|\partial \Lambda^{c}\right|\right) \sum_{\mathscr{L} \in \operatorname{CAN}_{q}^{N}(\Lambda)} \exp \left(-\sum_{i} \Phi^{*}\left(\Gamma_{i}\right)\right) \\
& \cdot \prod_{j} \exp \left(-a_{q\left(W_{j}\right)}\left|W_{j}\right|\right),
\end{aligned}
$$

$$
\Phi^{*}(\Gamma)=\Phi(\Gamma)-\left(h_{0}-e_{q}+2 C\right)|\operatorname{supp} \Gamma|
$$

and where we used the inequality [see also (1.43)]

$$
\sum_{i}\left|\partial W_{j}^{c}\right| \leqq 2 \sum_{i}\left|\operatorname{supp} \Gamma_{i}\right|+\left|\partial \Lambda^{c}\right|
$$

Now, a suitable factorization of contours is needed such that Lemma 2.3 could be applied to the estimate (3.9): consider the equivalency $\Gamma^{q} \sim \tilde{\Gamma}^{q}$ iff $\operatorname{supp} \Gamma^{q}=\operatorname{supp} \tilde{\Gamma}^{q}$ and $\operatorname{int}_{q^{\prime}} \Gamma^{q}=\operatorname{int}_{q^{\prime}} \tilde{\Gamma}^{q}$ for each nonstable $q^{\prime}$. Each equivalency class $\left[\Gamma^{q}\right]$ we identify with a contour in the sense of Sect. 2.3. Put

$$
\Phi^{*}\left(\left[\Gamma^{q}\right]=-\log \sum_{\tilde{\Gamma}^{q} \in\left[\Gamma^{q}\right]} \exp \left(-\Phi^{*}\left(\tilde{\Gamma}^{q}\right)\right) .\right.
$$

Define the free energy $s_{q}^{*}$ according to Lemma 2.3. It is easy to see [from (2.26)] that for a large $\tau=\tau(\alpha)$ we have, instead of (2.33), a stronger inequality

$$
s_{q}^{*}<(1-\alpha) a_{q}
$$

for any unstable $q$. 
Return now to (3.9). Using Lemma 2.3 and (3.13) we get

$$
\begin{aligned}
\mathbf{Z}_{q}^{N}(\Lambda, H) \leqq & \exp \left(-h_{0}|\Lambda|+C\left|\partial \Lambda^{c}\right|-\alpha a N\right) \\
& \cdot \sum_{[\mathscr{L}] \in\left[\operatorname{CAN}_{q}^{N}(\Lambda)\right]} \exp \left(-\sum_{i} \Phi^{*}\left(\left[\Gamma^{q}\right]\right) \prod_{j} \exp \left((\alpha-1) a_{q\left(W_{j}\right)}\left|W_{j}\right|\right)\right.
\end{aligned}
$$

(by $[\mathscr{L}],\left[\mathrm{CAN}_{q}^{N}(\Lambda)\right]$ we denote the equivalence classes, corresponding to the equivalency classes $\left[\Gamma^{q}\right]$ )

$$
\leqq \exp \left(-h_{0}|\Lambda|+2 C\left|\partial \Lambda^{c}\right|-\alpha a N\right) \text {. q.e.d. }
$$

\subsection{General Boundary Conditions}

It is a time now to drop the assumption about the special boundary conditions, used in the definitions of partition functions $\mathbf{Z}_{q}(\Lambda, H), \mathbf{Z}(\Gamma, H)$ throughout Sects. 1 and 3.1, and to consider a general partition function

$$
\mathbf{Z}\left(\Lambda, x_{\Lambda^{c}}, H\right)=\sum_{x_{\Lambda} \in S^{\Lambda}} \exp \left(-H\left(x_{\Lambda} \mid x_{\Lambda^{c}}\right),\right.
$$

where $x_{\Lambda^{c}}$ is an arbitrary configuration on $\Lambda^{c}$.

Note. We return to the original form (1.2) of $H\left(x_{\Lambda} \mid x_{\Lambda^{c}}\right)$ if needed. Also, we will not omit the third term of (1.7) if this expression is used.

Definition 1. Let $\Lambda$ be a simply connected set. Given any configuration $x_{\Lambda} \in S^{\Lambda}$ and any boundary condition $x_{\Lambda^{c}}$, we say that a point $t \in \Lambda$ is an "inner" point of $x_{\Lambda}$ if there is a simply connected set $\tilde{\Lambda} \subset \Lambda$ such that $\tilde{\Lambda} \ni t$ and all points of $\partial \tilde{\Lambda}$ are $q$-correct points of $x_{\Lambda} \cup x_{\Lambda^{c}}$ for some (stable or nonstable) $q$.

Definition 2. Decompose the set of all inner points into the components. Clearly, any such component $C$ is simply connected and there is some $q(=q(C))$ such that $x_{\Lambda}$ has, on $\partial C$, a constant value $q$ and all the contours of $\left(x_{\Lambda}\right)_{C} \cup\left(x_{q}\right)_{C^{c}}$ have a distance at least 2 from $C^{c}$. We say that $t \in \Lambda$ is a stable point of $x_{\Lambda}$ (at the condition $\left.x_{\Lambda^{c}}\right)$ if $t$ is a stable point of some $\left(x_{\Lambda}\right)_{C}$, in the sense of Definition 3 of 3.1 . We denote by $U(=U(t, x, \Lambda))$ the component of the stable domain of $\left(x_{\Lambda}\right)_{C}$, which contains $t$.

Theorem. Let $0<\alpha<1$. Suppose that $\tau$ [in (1.9)] is sufficiently large. Then there is a constant $K$ such that for each finite simply connected set $\Lambda \subset \mathbb{Z}^{v}$ and for each boundary condition $x_{\Lambda^{c}}$ the following estimate holds: Denote by $X_{x_{\Lambda^{c}}}^{N}(\Lambda)$ the set of all $x_{\Lambda} \in S^{\Lambda}$ which have at least $N$ unstable points. Denote by

$$
\begin{gathered}
\mathbf{Z}^{N}\left(\Lambda, x_{\Lambda^{c}}, H\right)=\sum_{x_{\Lambda} \in X_{x^{c}}^{N}(\Lambda)} \exp \left(-H\left(x_{\Lambda} \mid x_{\Lambda^{c}}\right)\right), \\
P^{N}\left(\Lambda, x_{\Lambda^{c}}, H\right)=\mathbf{Z}^{N}\left(\Lambda, x_{\Lambda^{c}}, H\right) \mathbf{Z}\left(\Lambda, x_{\Lambda^{c}}, H\right)^{-1} .
\end{gathered}
$$

Then, for any $N \in \mathbb{N}$,

$$
P^{N}\left(\Lambda, x_{\Lambda^{c}}, H\right) \leqq \exp (-\alpha a N) \exp \left(K\left|\partial \Lambda^{c}\right|\right) .
$$

Corollary. Let $\left\{\Lambda_{i}\right\}$ be a sequence of simple connected finite subsets of $\mathbb{Z}^{v}$ such that $\Lambda_{i} \uparrow \mathbb{Z}^{v}$ in the Van Hove sense. Let $\left\{x_{\Lambda_{i}^{c}}\right\}$ be any sequence of boundary conditions. Denote by $P_{x_{A^{c}}}$ the Gibbs state on $S^{\Lambda}$ corresponding to the boundary condition $x_{\Lambda^{c}}$. 
Suppose that a limit Gibbs state, defined for each cylindrical set $A \subset S^{\tilde{\Lambda}}, \tilde{\Lambda}$ finite, as

$$
P(A)=\lim _{i} P_{x_{\Lambda_{i}^{c}}^{c}}(A)
$$

exists. Suppose moreover that $P$ is a translation invariant Gibbs state. Then $P$ can be decomposed into the extremal Gibbs states constructed, for various stable $q$, in Sect. 1 .

Proof of Corollary. We will prove the following statement only (the rest of the proof is a consequence of standard methods of the theory of limit Gibbs states):

Lemma. Let $\Lambda$ be a finite simple connected subset of $\mathbb{Z}^{v}$. Let $x_{\Lambda}$ and $x_{\Lambda^{c}}$ be arbitrary configurations on $\Lambda$ respectively $\Lambda^{c}$. Recall the notion of the stable component $U$ containing a given point $t \in \Lambda$ (Definition 2$)$. For any stable q, any simply connected finite $\Lambda$ and any integer $d$, consider the event

$$
\Omega_{\Lambda}(t, d, q)=\left\{x: \operatorname{dist}\left(t, U^{c}\right) \leqq d, q(U)=q\right\},
$$

where $q(U)$ denotes the value of $x$ on $\partial U$. Consider further the events

$$
\tilde{\Omega}_{\Lambda}(t, d, q)=\bigcap_{\tilde{\Lambda}: \tilde{\Lambda} \supset \Lambda} \Omega_{\tilde{\Lambda}}(t, d, q) .
$$

Then for any integer $d$, any stable $q$ and any simple connected $\Lambda$,

$$
P\left(\tilde{\Omega}_{\Lambda}(t, d, q)\right)=0 \text {. }
$$

Note. Therefore, for each $t \in \mathbb{Z}^{v}$, each simply connected finite $\Lambda$ and each integer $d$ we can find, with probability 1 , some $\widetilde{\Lambda} \supset \Lambda$ and some simply connected set $U(t, x, \Lambda)$ such that $\operatorname{dist}\left(t, U^{c}\right)>d$ and the restriction of the given configuration to $\partial U$ has a constant value, equal to some stable $q$. Now it suffices to use the decay of correlations in any of the Gibbs fields of Sect. 1.

Proof of Lemma. First notice the following obvious consequence of (3.18): Suppose that $Q$ is a probability on $S^{\mathbb{Z}^{v}}$ such that all its conditional probabilities on $S^{\Lambda}$, for any $x_{\Lambda^{c}}$, are equal to the Gibbsian conditional probabilities. Denote by $X^{N}(\Lambda)$ the event $\bigcup_{x_{\Lambda^{c}}} X_{x_{\Lambda^{c}}}^{N}(\Lambda) \subset S^{\mathbb{Z}^{v}}$. Then

$$
Q\left(X^{N}(\Lambda)\right) \leqq \exp (-\alpha a N) \exp \left(K\left|\partial \Lambda^{c}\right|\right) .
$$

Suppose, on the contrary to (3.22) that for some $t, d, q, \Lambda$,

$$
P\left(\tilde{\Omega}_{\Lambda}(t, d, q)>\omega \text { for some } \omega>0 .\right.
$$

Using the translation invariancy of $P$ and the relation

$$
\Omega_{\tilde{\Lambda}}(t, d, q) \supset \tilde{\Omega}_{\Lambda}(t, d, q), \quad \tilde{\Lambda} \supset \Lambda,
$$

we obtain, for any sufficiently large cube $W \subset \mathbb{Z}^{v}$ the inequality

$$
\sum_{t \in W} P\left(\Omega_{W}(t, d, q)\right)>\omega|W| .
$$

But $\sum_{t \in W} P\left(\Omega_{W}(t, d, q)\right)$ is the mathematical expectation of the number $M$ of those $t \in \Lambda$ for which $x$ belongs to $\Omega_{W}(t, d, q)$. Estimate $M$ by using the property that

$$
x \in \Omega_{W}(t, d, q)=>\|t-s\| \leqq d
$$


for some unstable point $S$ of $x_{A}$ (with respect to $x_{\Lambda^{c}}$ ). Clearly, then

$$
M \leqq(2 d)^{v} N
$$

where $N$ is the number of unstable points. Thus, the expectation of $N$ (in $P$ ) would be greater than $(2 d)^{-v} \alpha|W|$ which contradicts (3.23) if $W$ is large. This proves (3.22).

Proof of Theorem. We will relate (3.18) to the basic estimate (3.5). Recall the notion of an inner point (Definition 1). Given $x_{A}$ and $x_{\Lambda^{c}}$ denote by $x_{\Lambda}^{\text {out }}$ the restriction of $x_{\Lambda}$ to the set Out $x_{\Lambda}$ of all noninner points of $x_{\Lambda}$. Denote by $W$ the set $\Lambda \backslash$ Out $x_{\Lambda}$. Denote by $W_{q}$ the union of all the components $C$ of $W$ which are $q$-components (i.e. such that $x_{t}=q$ for $\left.t \in \partial C\right)$. We get the relation

$$
\mathbf{Z}^{N}\left(\Lambda, x_{\Lambda^{c}}, H\right)=\sum_{\left\{x_{\Lambda}^{\text {out }}\right\}} \exp \left(-H\left(x_{\Lambda}^{\text {out }} \mid x_{W} \cup x_{\Lambda^{c}}\right)\right) \sum_{\left\{N_{q}\right\}} \prod_{q} \mathbf{Z}_{q}^{N_{q}}\left(W_{q}, H\right),
$$

where the first sum is taken over all possible $\left\{x_{\Lambda}^{\text {out }}\right\}$ and the second sum is taken over all $\left\{N_{q}\right\}$ such that $N=\sum_{q \in Q} N_{q}$. Using (3.5) in (3.26) we get

$$
\mathbf{Z}^{N}\left(\Lambda, x_{\Lambda^{c}}, H\right) \leqq n^{*} \sum_{\left\{x^{\text {out }}\right\}} \exp \left(-H\left(x_{\Lambda}^{\text {out }} \mid x_{W} \cup x_{\Lambda^{c}}\right)\right) \exp \left(-h_{0}|\Lambda|-\alpha a N+\sum_{q} R_{q}\left(W_{q}\right)\right),
$$

where $n^{*}$ is the number of possible partitionings $\left\{N_{q}\right\}$ of $N$, and where $R_{q}(W)$ is given by the formula

$$
R_{q}(W)=C\left|\partial W^{c}\right|+\sum_{A \not \subset W} \frac{\left|A \cap W^{c}\right|}{|A|} \Phi_{A}\left(\left(x_{q}\right)_{A}\right),
$$

[see (1.7)]. Notice that

$$
\sum_{A \nsubseteq W} \frac{\left|A \cap W^{c}\right|}{|A|} \Phi_{A}\left(\left(x_{q}\right)_{A}\right)<C_{2}\left|\partial W^{c}\right| .
$$

for some universal constant $C_{2}$. Notice also [this is a similar inequality as (1.43), (2.37)] that

$$
\sum_{q}\left|\partial W_{q}^{c}\right| \leqq \mid \text { Out } x_{\Lambda}|+| \partial \Lambda \mid
$$

$C_{4}=C_{3}$. We obtain the inequality, with $C_{3}=C+C_{2}$,

$$
\begin{aligned}
\mathbf{Z}^{N}\left(\Lambda, x_{\Lambda^{c}}, H\right) \leqq & n^{*} \sum_{\left\{x_{\Lambda}^{\text {out }}\right\}} \exp \left(-H\left(x_{\Lambda}^{\text {out }} \mid x_{W \cup \Lambda^{c}}\right)\right) \\
& \cdot \exp \left(-h_{0}|\Lambda|-\alpha a N+C_{3}|\partial \Lambda|+C_{4}\left|\mathrm{Out}_{x_{\Lambda}}\right|\right) .
\end{aligned}
$$

Because no point of Out $x_{A}$ is correct we can use the Peierls condition (1.9) and the boundedness of all interactions $\Phi_{A}$ to find another universal constant $C_{5}$ such that for each $x$,

$$
\left.H\left(x_{\Lambda}^{\text {out }} \mid x_{W \cup \Lambda^{c}}\right)\right) \geqq \tau \mid \text { Out } x_{\Lambda}\left|-C_{5}\right| \partial \Lambda \mid .
$$

At last, choose another constant $C_{6}$ such that $\exp \left(C_{6}|T|\right)$ would be an upper bound for the number of all possible $x_{\Lambda}^{\text {out }}$ with the same Out $x_{\Lambda}=T$. Substituting this into (3.31), 


$$
\begin{gathered}
\mathbf{Z}^{N}\left(\Lambda, x_{\Lambda^{c}}, H\right) \leqq n^{*} \sum_{\{\text {Out }\}} \exp \left(-\tau \mid \text { Out } x_{\Lambda}\left|+c_{7}\right| \text { Out } x_{\Lambda}\left|+c_{8}\right| \partial \Lambda^{c} \mid\right) \\
\cdot \exp \left(-h_{0}|\Lambda|-\alpha a N\right)
\end{gathered}
$$

where $C_{7}=C_{6}+C_{4}, C_{8}=C_{5}+C_{3}$, and the sum is taken over all possible Out $\equiv$ Out $x_{\Lambda}$. Notice that Out $x_{\Lambda} \cup \partial \Lambda$ is a connected set. It follows from this observation and (3.33) that for a large $\tau$, there is another universal constant $K$ such that

$$
\mathbf{Z}^{N}\left(\Lambda, x_{\Lambda^{c}}, H\right) \leqq n^{*} \exp \left(K\left|\partial \Lambda^{c}\right|-h_{0}|\Lambda|-\alpha a N\right)
$$

To remove $n^{*}$ notice that $n^{*}=o\left(q^{N}\right), N \rightarrow \infty$, for any $q>1$. Therefore, for any $\alpha^{\prime}<\alpha$, there is a suitable $K^{\prime}$ such that

$$
\mathbf{Z}^{N}\left(\Lambda, x_{\Lambda^{c}}, H\right) \leqq \exp \left(K^{\prime}\left|\partial \Lambda^{c}\right|-h_{0}|\Lambda|-\alpha^{\prime} a N\right) .
$$

On the other hand it is clear from the boundedness of all $\Phi_{A}$ that for each stable $q$, there is another constant $L$ such that

$$
\mathbf{Z}\left(\Lambda, x_{\Lambda^{c}}, H\right) \geqq \exp \left(L\left|\partial \Lambda^{c}\right|\right) \mathbf{Z}_{q}(\Lambda, H),
$$

i.e., by Theorem 1.7, there is a constant $L^{\prime}$ such that for each $\Lambda$ and each $x_{\Lambda^{c}}$ the following inequality holds:

$$
\mathbf{Z}\left(\Lambda, x_{\Lambda^{c}}, H\right) \geqq \exp \left(L^{\prime}\left|\partial \Lambda^{c}\right|-h_{0}|\Lambda|\right) .
$$

It is clear that (3.35) and (3.36) give the desired estimate (3.18). q.e.d.

Acknowledgement. It is a pleasure to thank D. Preiss, R. Kotecký and other participants of our Prague seminar for stimulating conversations on the subject. A general result on the completeness of the phase diagram was obtained independently, using a different method, by D. Preiss (unpublished). For another approach to PS theory (closer to [2]) see [7].

\section{References}

1. Minlos, R.A., Sinai, Ya.G.: Tr. Mosk. Math. Obshch. 19, (1968), in Russian

2. Pirogov, S.A., Sinai, Ya.G.: Teor. Mat. Fiz. 25, 358-369 (1975), in Russian; English translation: Phase diagrams of classical lattice systems. Theor. Math. Phys. 25, 1185-1192 (1975); and Teor. Mat. Fiz. 26, 61-76 (1976), in Russian; English translation: Phase diagrams of classical lattice systems. Continuation. Theor. Math. Phys. 26, 39-49 (1976)

3. Sinai, Ya.G.: Theory of phase transitions: rigorous results. Oxford, New York: Pergamon Press, 1982

4. Martirosian, D.G.: The problem of an upper estimate of the number of periodic Gibbs states for the model of a latticed gas. Usp. Mat. Nauk 30, 181 (1975)

5. Malyshev, V.A.: Usp. Mat. Nauk 35, 212 (1980), in Russian; English translation: Cluster expansions in lattice models of statistical physics and the quantum theory of fields. Russ. Math. Surv. 35, 1-62 (1980)

6. Bricmont, J., Lebowitz, J.L., P fister, C.-E.: Low temperature expansion for continuous-spin Ising models. Commun. Math. Phys. 78, 117-135 (1980)

7. Kotecký, R., Preiss, D.: An inductive approach to PS theory. Proc. Winter School on Abstract Analysis 1983. Suppl. Ai Rend. Del Circ. Mat. Di Palermo (1983)

Communicated by Ya. G. Sinai

Received October 25, 1983 
